\title{
Hybrid analytic-numeric method for light through a bounded planar dielectric domain
}

\begin{abstract}
(3)
University of Twente The Netherlands
\end{abstract}

\section{J. B. Nicolau and E. van Groesen}

Dept. of Applied Mathematics, MESA ${ }^{+}$Research Institute, University of Twente, P.O. Box 217, 7500 AE Enschede, The Netherlands.

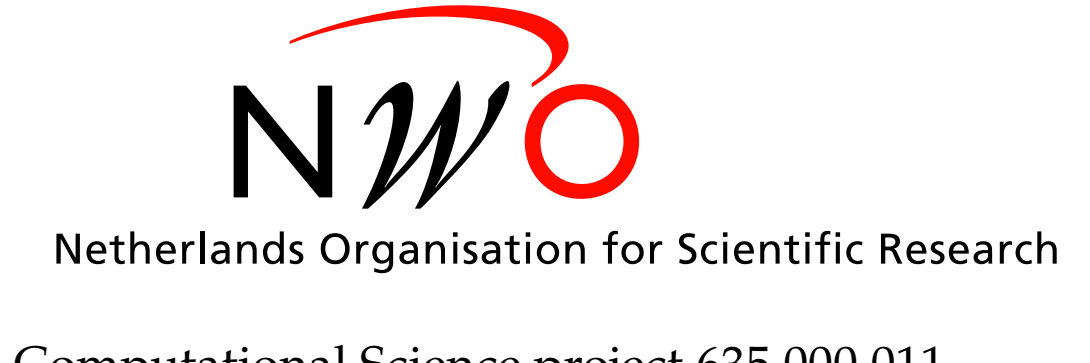

Computational Science project 635.000.011

We present a hybrid analytic-numeric method to calculate the transmission and reflection of light that is fluxed into a bounded complicated optical structure surrounded by air. The solution is obtained by numerical calculations inside a square containing the structure and by analytical calculations outside the square. For solving the 2D Helmholtz equation we formulate Transparent-Influx Boundary Conditions (TIBC's) on the boundaries of the square; these are incorporated into a variational formulation of the Helmholtz equation to obtain a FEM-implementation for the interior calculations.

\section{Modelling problem and general approach}

On the plane, when restricting to TE-polarization, the propagation of time-harmonic light is described by the scalar Helmholtz Equation (HE) for the principal component $E$ of the electric field perpendicular to the plane,

$$
\Delta E+k^{2} E=0,
$$

where $k(x, z)=k_{0} n(x, z)$, with $k_{0}$ the free-space wavenumber, $n(x, z)$ is the refractive index of the media characterizing the geometry of the device, and $k_{0}=\frac{2 \pi}{\lambda}, \lambda$ is the vacuum wavelength. Our approach for solving the Helmholtz problem is as follows:

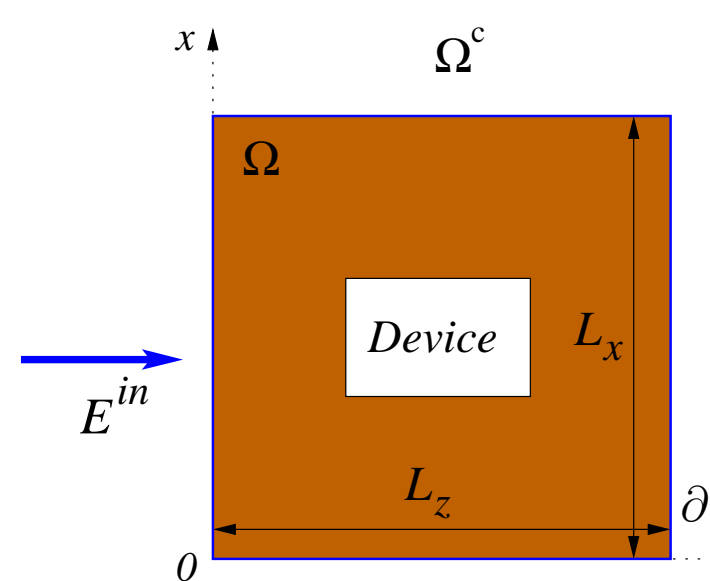

\section{- given influx in exterior: $E^{i n}$}

- formulate TIBC's on $\partial \Omega$, which will include influx information $f=\left.E^{i n}\right|_{\partial \Omega}$

- calculate numerically the solution $E_{\Omega}$ in $\Omega$ with TIBC's on $\partial \Omega$

- determine the boundary value and calculate the $E^{\text {out }}$ from $\left.E_{\Omega}\right|_{\partial \Omega}-\left.E^{i n}\right|_{\partial \Omega}$

- $E_{\Omega}$ and $E^{e x t}=E^{i n}+E^{o u t}$ in $\Omega^{c}$ satisfy the correct continuity conditions on $\partial \Omega \Rightarrow$ solution on the whole domain.

\section{Transparent-influx boundary conditions (TIBC's)}

- An incoming field is assumed from the left $\Rightarrow E^{i n}=f \quad$ and $\quad \partial_{n} E^{i n}=F \quad$ at $\partial \Omega$

- Interface conditions $\Rightarrow E_{\Omega}=E^{e x t}$ and $\partial_{n} E_{\Omega}=\partial_{n} E^{e x t}$ at $\partial \Omega$

- Solution in the exterior $\Rightarrow E^{e x t}=E^{\text {in }}+E^{\text {out }}$

- Dirichlet-to-Neumann operators (DtN): given a Dirichlet data $g$ at $\partial \Omega$

$$
\begin{array}{ll}
D^{+}(g)=\left.\partial_{n} E\right|_{\partial \Omega} & E \text { outgoing solution (HE) with }\left.E\right|_{\partial \Omega}=g \\
D^{-}(g)=\left.\partial_{n} E\right|_{\partial \Omega} & E \text { incoming solution (HE) with }\left.E\right|_{\partial \Omega}=g
\end{array}
$$$$
D^{-}(g)=\left.\partial_{n} E\right|_{\partial \Omega} \quad E \text { incoming solution (HE) with }\left.E\right|_{\partial \Omega}=g
$$

- Analytical expressions for the DtN operators can be obtained via plane wave decomposition

- For the square with influx though the western side, we find as boundary conditions:

$$
\begin{aligned}
\partial_{n} E_{\Omega}-D_{W}^{+}\left(\left.E_{\Omega}\right|_{\partial \Omega}\right) & =D^{-}(f)-D^{+}(f) & & \text { on } \partial \Omega_{W} \text { with } f=\left.E^{i n}\right|_{\partial \Omega} \\
\partial_{n} E_{\Omega}-D_{\gamma}^{+}\left(\left.E_{\Omega}\right|_{\partial \Omega}\right) & =0 & & \text { on } \partial \Omega_{\gamma} \text { for } \gamma=N, E, S
\end{aligned}
$$

\section{Numerical implementation}

We formulate a discretization of the TIBC's as follows:

- On the western side we have boundary function $g$ on the interval $\left[0, L_{x}\right]$. We present this function by

$$
g(x)=\sum_{m=-\infty}^{\infty} \widehat{g}(m) e^{i\left[m h_{x} x\right]}, \text { with } h_{x}=\frac{2 \pi}{\alpha L_{x}} \text { and } \widehat{g}(m)=\frac{1}{\alpha L_{x}} \int_{0}^{L_{x}} g(x) e^{i\left[-m h_{x} x\right]} d x
$$

- Taking $\alpha=1 \Rightarrow g$ is represented by a periodic series, with period which is precisely the length of the side.

- Taking $\alpha>1 \Rightarrow g$ is zero outside the basic interval.

- If the given function $g$ at the western boundary corresponds to an influx field, this field is given by

$$
E^{i n}(x, z)=\sum_{m=-\infty}^{\infty} \widehat{g_{i n}}(m) e^{i\left[m h_{x} x+\beta\left(m h_{x}\right) z\right]} \quad \text { for } z<0 \text {, where }\left\{\beta\left(m h_{x}\right)\right\}^{2}+\left\{m h_{x}\right\}^{2}=k_{0}^{2} .
$$

- The corresponding DtN operators : $D_{W}^{+}\left(\left.E^{i n}\right|_{\partial \Omega}\right)=-D_{W}^{-}\left(E^{i n} \mid \partial \Omega\right)=\sum_{m=-\infty}^{\infty} i \beta\left(m h_{x}\right) \widehat{g_{i n}}(m) e^{i\left[m h_{x} x\right]}$. Remark: The outgoing fields at the boundaries can be found in a similar manner.

\section{FEM implementation}

- Variational form of the Helmholtz equation:

$$
\mathcal{L}(V, E):=\underbrace{\iint_{\Omega} \nabla V \cdot \nabla E_{\Omega}-k_{\Omega}^{2} V E_{\Omega} \mathrm{d} s}_{\text {Interior }}-\underbrace{\int_{\partial \Omega} V \partial_{n} E^{e x t} \mathrm{~d} l}_{\text {Boundary }}=0, \forall V \in H^{1}(\Omega)
$$

with $\quad \int_{\partial \Omega} V \partial_{n} E^{e x t} \mathrm{~d} l=\int_{\partial \Omega} V\left\{D^{+}\left(\left.E_{\Omega}\right|_{\partial \Omega}\right)+D^{-}\left(E^{i n} \mid \partial \Omega\right)-D^{+}\left(E^{i n} \mid \partial \Omega\right)\right\} \mathrm{d} l$.
Challenge: Boundary operator discretization

- The obtained analytical expressions for the DtN operators can be incorporated into the variational form of the HE.

$\Rightarrow$ Discrete form of the DtN operator: $\int_{0}^{L_{x}} V D_{W}^{+}\left(g_{W}\right) \mathrm{d} l \cong \frac{1}{\alpha L_{x}} \sum_{p . q}^{N} v_{p} g_{q}\left(\sum_{m} i \beta\left(m h_{x}\right) \widehat{\tau}_{q m} \tau_{p m}\right)$

with $\quad \widehat{\tau}_{q m}=\int_{0}^{L_{x}} \phi_{q}(x, 0) e^{i\left[-m h_{x} x\right]} \mathrm{d} x \quad$ and $\quad \tau_{p m}=\int_{0}^{L_{x}} \phi_{p}(x, 0) e^{i\left[m h_{x} x\right]} \mathrm{d} x$.

Note that the range of $m$ is now finite for numerical purposes and $\phi_{j}$ are the piecewise linear basis functions.

\section{Numerical results}

Interior domain

Parameters: computational window of $[0,10] \mu m \times[0,10] \mu m, \lambda=1 \mu m$.

- Error analysis: propagation of a Gaussian beam in free space

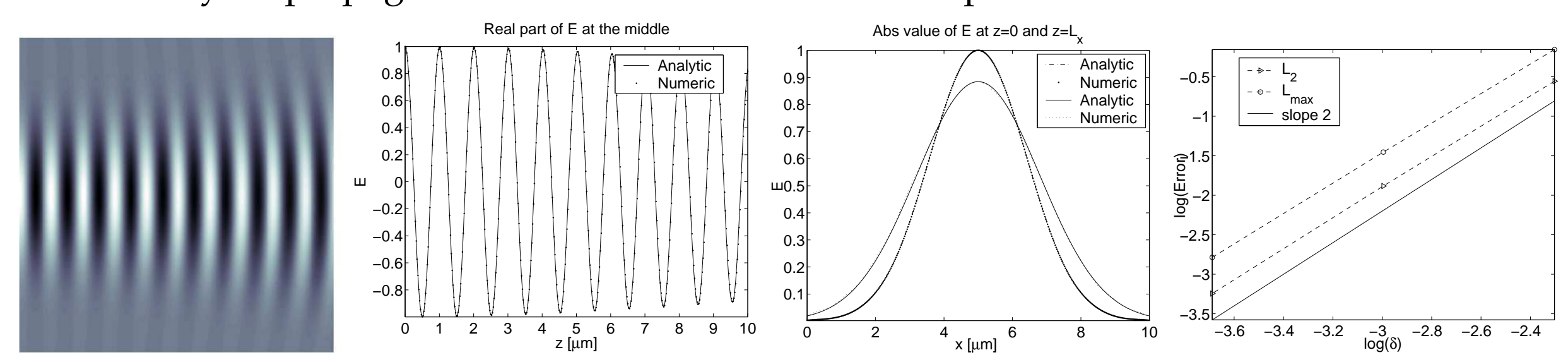

- Inhomogeneous interior: $n_{d}=3.25$
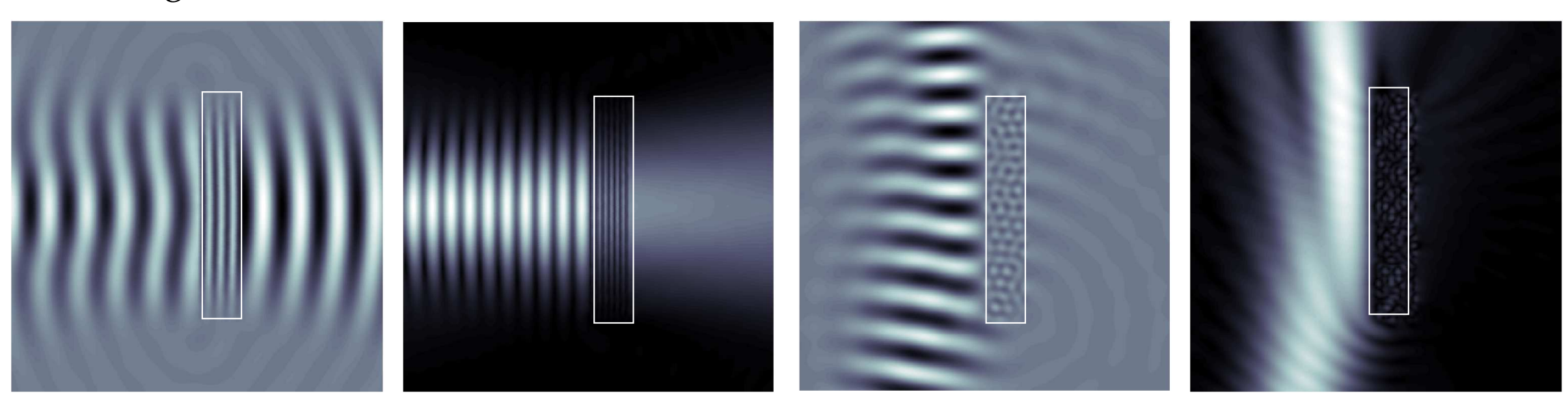

Exterior: hybrid analytic-numeric method

The Hybrid Window (HW): Interior domain $\Omega$ (FEM solution) + a region on which the solution is extended in the exterior.
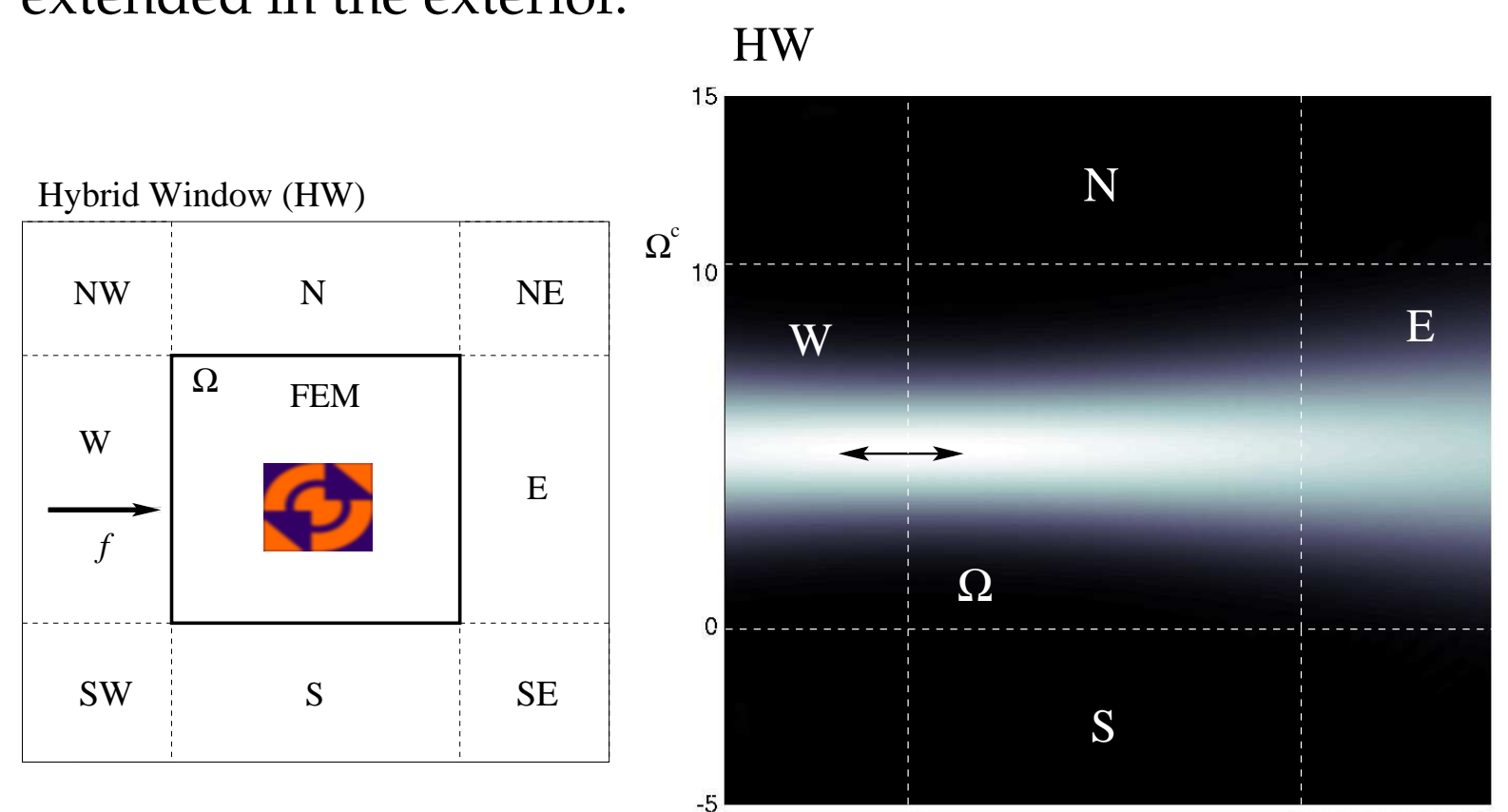

(a) $\mathrm{HW}$

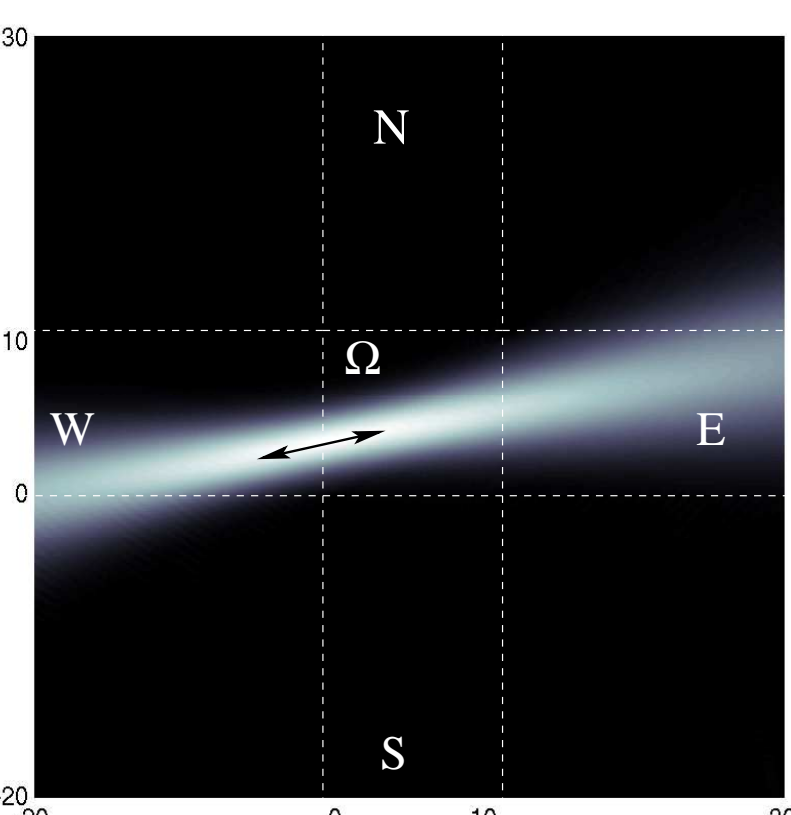

(b)

(a) A horizontally centered Gaussian beam with $\mathrm{HW}=[-5,15] \mu \mathrm{m} \times[-5,15] \mu \mathrm{m}$ (b) An obliquely Gaussian beam with $\mathrm{HW}=[-20,30] \mu m \times[-20,30] \mu m$.

\section{Conclusions}

- We have developed well posed boundary conditions (TIBC's) for 2D Helmholtz problems in optics

- The boundary conditions depend only on the behavior of the solution in the exterior domain

- Implementation of those boundary conditions can be done in any numerical method

- The boundary conditions are formulated here for harmonic problems (TE-modes). Extension to TM-case is immediate.

\section{References}

[1] D. Givoli, I. Comput. Phys. 94, 1 (1991).

[2] E. van Groesen, Variational modelling for integrated optical devices - Proc. 4th IMACS-symposium on Mathematical Modelling, TU Vienna, 76 (2003).

[3] S. Choudhary and L. B. Felsen, Proc. IEEE 62, 1530 (1974).

[4] J. B. Nicolau and E. van Groesen, Hybrid analytic-numeric method for light through a bounded planar dielectric domain, accepted for publication in JNOPM, March 2005. 